\title{
Desenvolvimento de trombose em pacientes com infecção por SARS-CoV-2
}

\author{
Development of thrombosis in patients with SARS-CoV-2 infection
}

Desarrollo de trombosis en pacientes con infección por SARS-CoV-2

Lennara Pereira Mota ORCID: https://orcid.org/0000-0002-2629-6634 Centro Universitário Maurício de Nassau, Brasil E-mail: lennaramota@hotmail.com

Maria Vitalina Alves de Sousa ORCID: https://orcid.org/0000-0003-4448-2489 Centro Universitário INTA, Brasil E-mail: enf.vitalinaalves@gmail.com

José Nilton de Araújo Gonçalves ORCID: https://orcid.org/0000-0002-1578-3656

Universidade Federal de Mato Grosso, Brasil E-mail: josenilton.ifpi@hotmail.com

Nahadja Tahaynara Barros Leal ORCID: https://orcid.org/0000-0002-4833-9914 Universidade Federal de Campina Grande, Brasil E-mail: nahadja@gmail.com

Loisláyne Barros Leal ORCID: https://orcid.org/0000-0002-1294-686X Universidade Federal do Piauí, Brasil E-mail: loislaynebarros@gmail.com

Ana Luiza Barbosa Negreiros ORCID: https://orcid.org/0000-0002-8313-0403 Universidade Federal do Piaú, Brasil

E-mail: analuiza.negreiros@ hotmail.com

Danyele Holanda da Silva ORCID: https://orcid.org/0000-0001-7517-5890 UNINASSAU, Brasil

E-mail: dan_sil_holanda@hotmail.com

Ana Alinne Gomes Da Penha

ORCID: https://orcid.org/0000-0001-9253-1199 Universidade Regional do Cariri, Brasil E-mail: anaalinne.nurse@gmail.com

Nágila Silva Alves

ORCID: https://orcid.org/0000-0002-1618-8111 Centro Universitário Santo Agostinho, Brasil E-mail: nglarraial@gmail.com

Priscila de Freitas Ferreira ORCID: https://orcid.org/0000-0002-8716-7801

Universidade Federal de Juiz de Fora, Brasil

E-mail: prifferreira84@gmail.com

Gersilane Lima Leal

ORCID: https://orcid.org/0000-0001-5272-1860 Universidade Estadual do Piauí, Brasil E-mail: gersilane.leal19@gmail.com

Francisco De Assis Da Silva Sousa

ORCID: https://orcid.org/0000-0002-9665-5284

Hospital Regional Do Sertão Central, Brasil E-mail: fassis90assis@gmail.com

Francilene Vieira Da Silva Freitas ORCID: https://orcid.org/0000-0003-3647-1587 UEMA - Universidade Estadual do Maranhão, Brasil E-mail: francilenev@gmail.com

Eudes Rufino da Silveira Filho

ORCID: https://orcid.org/0000-0003-0360-1322 Centro Universitário UNINOVAFAPI, Brasil E-mail: personalgigaeudes@gmail.com

Jaqueline Dantas da Costa

ORCID: https://orcid.org/0000-0003-2570-1949 Centro Universitário UNINOVAFAPI, Brasil E-mail: jaquedc87@gmail.com 


\author{
Mayara Madeira de Carvalho \\ ORCID: https://orcid.org/0000-0002-0768-3874 \\ Centro de Ensino Unificado de Teresina, Brasil \\ E-mail: mayangel1000@ @otmail.com \\ Maira Gislany de Castro Pereira \\ ORCID: https://orcid.org/0000-0002-8993-9510 \\ Universidade Estadual do Piauí, Brasil \\ E-mail: maira_gislanny22@ @otmail.com \\ Stefânia Araújo Pereira \\ ORCID: https://orcid.org/0000-0001-5288-8193 \\ Universidade Estadual do Piauí, Brasi \\ E-mail: stefania.rillys@gmail.com \\ Joice Mara Ferreira Dos Santos \\ ORCID: https://orcid.org/0000-0002-3231-750X \\ Associação de Ensino Superior do Piauí, Brasil \\ E-mail: joicinha2.2kinha@gmail.com
}

\begin{abstract}
Resumo
A pandemia causada pelo coronavírus SARS-CoV-2, além de possivelmente provocar lesão pulmonar alveolar e falência respiratória aguda, apresenta uma alta prevalência de doenças cardiovasculares, sobretudo tromboembolismo venoso (TEV). O trabalho tem por objetivo expor através da analise de artigos científicos as principais complicações do desenvolvimento de trombose em pacientes infectados pelo COVID-19. Trata-se de uma revisão bibliográfica de caráter qualitativo que se baseia na produção científica a partir de estudos já publicados entre os anos de 2019 a 2021 . Alguns estudos sugerem que pacientes com COVID-19 podem apresentar um estado de hipercoagulabilidade, em que ocorre um aumento dos níveis séricos de fator VIII, fator de Von Willebrand e fibrinogênio. A hipercoagulabilidade pode ter influencia no desenvolvimento de trombose venosa profunda dos membros inferiores e/ou embolia pulmonar nesses pacientes. A infecção pelo SARS-Cov-2 se apresenta com uma variedade de complicações, sendo frequentes os relatos de problemas cardiovasculares e a presença de um estado pró-trombótico, causado por mecanismos ainda não totalmente elucidados. As evidências sobre COVID-19 e sua interação com o sistema de coagulação e ativação plaquetária ainda não estão totalmente esclarecidos.
\end{abstract}

Palavras-chave: Covid-19; Síndrome respiratória; Coagulação; Tromboembolismo.

\begin{abstract}
The pandemic caused by the SARS-CoV-2 coronavirus, in addition to possibly causing alveolar lung injury and acute respiratory failure, has a high prevalence of cardiovascular diseases, especially venous thromboembolism (VTE). The work aims to expose, through the analysis of scientific articles, the main complications of the development of thrombosis in patients infected with COVID-19. This is a literature review of a qualitative nature based on scientific production based on studies already published between the years 2019 to 2021. Some studies suggest that patients with COVID-19 may present a state of hypercoagulability, in which a state of hypercoagulability occurs. increased serum factor VIII, von Willebrand factor and fibrinogen levels. Hypercoagulability may influence the development of deep venous thrombosis of the lower limbs and/or pulmonary embolism in these patients. SARS-Cov-2 infection presents with a variety of complications, with frequent reports of cardiovascular problems and the presence of a prothrombotic state, caused by mechanisms that have not yet been fully elucidated. The evidence about COVID-19 and its interaction with the coagulation and platelet activation system is not yet fully understood.
\end{abstract}

Keywords: Covid-19; Respiratory syndrome; Coagulation; Thromboembolism.

\title{
Resumen
}

La pandemia provocada por el coronavirus SARS-CoV-2, además de provocar posiblemente lesión pulmonar alveolar e insuficiencia respiratoria aguda, tiene una alta prevalencia de enfermedades cardiovasculares, especialmente tromboembolismo venoso (TEV). El trabajo tiene como objetivo exponer, a través del análisis de artículos científicos, las principales del complicaciones desarrollo de trombosis en pacientes infectados por COVID-19. Se trata de una revisión de la literatura de carácter cualitativo basada en la producción científica basada en estudios ya publicados entre los años 2019 a 2021. Algunos estudios sugieren que los pacientes con COVID-19 pueden presentar un estado de hipercoagulabilidad, en el que se produce un estado de hipercoagulabilidad aumentado. niveles séricos de factor VIII, factor von Willebrand y fibrinógeno. La hipercoagulabilidad puede influir en el desarrollo de trombosis venosa profunda de los miembros inferiores y / o embolia pulmonar en estos pacientes. La infección por SARS-Cov-2 se presenta con una variedad de complicaciones, con informes frecuentes de problemas cardiovasculares y la presencia de un estado protrombótico, causado por mecanismos que aún no se han dilucidado por completo. La evidencia sobre COVID-19 y su interacción con el sistema de coagulación y activación plaquetaria aún no se comprende completamente.

Palabras clave: COVID-19; Síndrome respiratorio; Coagulación; Tromboembolismo. 


\section{Introdução}

A COVID-19 é uma doença respiratória de origem viral que apresenta sintomas graves de síndrome respiratória aguda causada pelo SARS-CoV-2. Pacientes infectados possuem risco de desenvolver trombose dos seguimentos arteriais e venosos, em consequência do quadro inflamatório demasiado e de fenômenos hematológicos, tais como, ativação plaquetária, disfunção do endotélio e estase sanguínea (Roberto et al., 2020).

A pandemia causada pelo coronavírus SARS-CoV-2, além de possivelmente provocar lesão pulmonar alveolar e falência respiratória aguda, apresenta uma alta prevalência de doenças cardiovasculares, sobretudo tromboembolismo venoso (TEV). O aumento do risco parece estar geralmente associado à reação inflamatória intensa e a liberação exacerbada de citocinas, sobretudo interleucina 6. A coagulopatia é avaliada pelo aumento dos índices de fibrinogênio, D-dímero (DD), fator VIII e prolongamento do tempo de protrombina (TP) e do tempo de tromboplastina parcial ativada (TTPa), esses fatores estão associados a má evolução clínica e óbito desses pacientes (Rossi, 2020).

Um dos parâmetros alterados por pacientes infectados pelo SARS-CoV-2 é o D-dímero. Às vezes, o número de Ddímeros continua a aumentar, embora outros parâmetros que geralmente alteram nesta doença, como ferritina, LDH e proteína C, tendem a se normalizar. Quando isso acontece, o diagnóstico diferencial é de um possível Tromboembolismo pulmonar (EP) sendo esta uma complicação de COVID-19 (Franco-López et al., 2020).

A COVID-19 está relacionada aos eventos de coagulopatia mostrados, principalmente, em pacientes gravemente enfermos infectados com SARS-CoV-2 devido a fatores pró-coagulantes aumentados. Além disso, devido à tempestade de citocinas causada pelo vírus, o aumento da incidência de embolia pulmonar e trombose microvascular pulmonar estão relacionados à doença, por meio do recrutamento e ativação dos glóbulos brancos (Carvalho et al., 2020).

O mecanismo da coagulopatia que ocorre na COVID-19 é complexo, mas especula-se que a doença endotelial e a ativação plaquetária podem ser fatores importantes, levando ao dano direto às células endoteliais e ao desequilíbrio da resposta inflamatória, manifestada como trombose venosa, trombose arterial ou trombose microvascular. Dentre as principais condições de risco para complicações, os idosos são o consenso da maioria dos artigos, e a média de idade está entre 65 a 70 anos. Isso se deve ao envelhecimento do sistema imunológico e à associação entre idade avançada e coexistência de comorbidades (Tiburi et al., 2021).

O estado inflamatório intensificado acaba levando à estase sanguínea, ativação plaquetária e disfunção endotelial, aumentando a chance de trombose venosa e arterial. A coagulopatia na infecção grave por COVID-19 é semelhante à coagulopatia causada pela sepse, que se caracteriza por coagulação intravascular disseminada (CIVD) e microangiopatias trombóticas. Além disso, é importante notar que a hipoxemia secundária à lesão pulmonar causada por COVID-19 é um fator de risco para o desenvolvimento de trombose (Brandão et al., 2021)

Em consequência do alto risco de desenvolvimento de tromboembolismo venoso em pacientes com COVID-19, recomendam-se estratégias de prevenção não relacionadas ao uso de drogas. Estudos sugerem que a estratégia farmacológica que deve ser considerada é o uso de heparina não fracionada ou heparina de baixo peso molecular, atentando-se às suas contraindicações e a taxa de depuração de creatinina do paciente (De Freitas et al., 2021).

O trabalho tem por objetivo expor através da análise de artigos científicos as principais complicações no desenvolvimento de trombose em pacientes infectados pelo COVID-19.

\section{Métodos}

Trata-se de uma revisão bibliográfica de caráter qualitativo que se baseia na produção científica a partir de estudos já publicados. A busca pelos textos foi realizada a partir das seguintes palavras-chaves indexadas no DECS (Descritores em 
Ciências da Saúde): "Covid-19"; "Síndrome Respiratória"; "Coagulação" e "Tromboembolismo" na plataforma Scientific Electronic Library Online (SCIELO) e Biblioteca Virtual em Saúde (BVS).

Os critérios de inclusão foram pesquisas científicas publicadas de 2019 a 2021, publicados no idioma português, inglês e espanhol, que atendiam ao problema da pesquisa: Quais as principais complicações no desenvolvimento de trombose em pacientes infectados por SARS-CoV-2? Os critérios de exclusão foram trabalhos científicos com apenas resumos disponíveis, publicações duplicadas, artigos de relato de experiência, reflexivo, editoriais, comentários e cartas ao editor.

A partir do problema de pesquisa foram selecionados artigos publicados em periódicos nacionais e internacionais escolhidos a partir de levantamento realizado por meios dos descritores na biblioteca virtual Scientific Electronic Library Online, Biblioteca Virtual em Saúde (BVS) e dissertações e teses.

Os artigos foram selecionados para leitura e foram incluídos na análise quantitativa artigos que se enquadravam no tema proposto do trabalho.

\section{Resultados e Discussão}

A Figura 1 ilustra os resultados deste trabalho que foram obtidos através da analise quantitativa de publicações sobre as principais complicações no desenvolvimento de trombose em pacientes infectados por SARS-CoV-2 nas bases de dados Scientific Electronic Library Online e Biblioteca Virtual em Saúde (BVS). 
Figura 1: Fluxograma que apresenta o processo de seleção das publicações sobre o desenvolvimento de trombose em pacientes infectados por SARS-CoV-2, Teresina, Brasil, 2021.

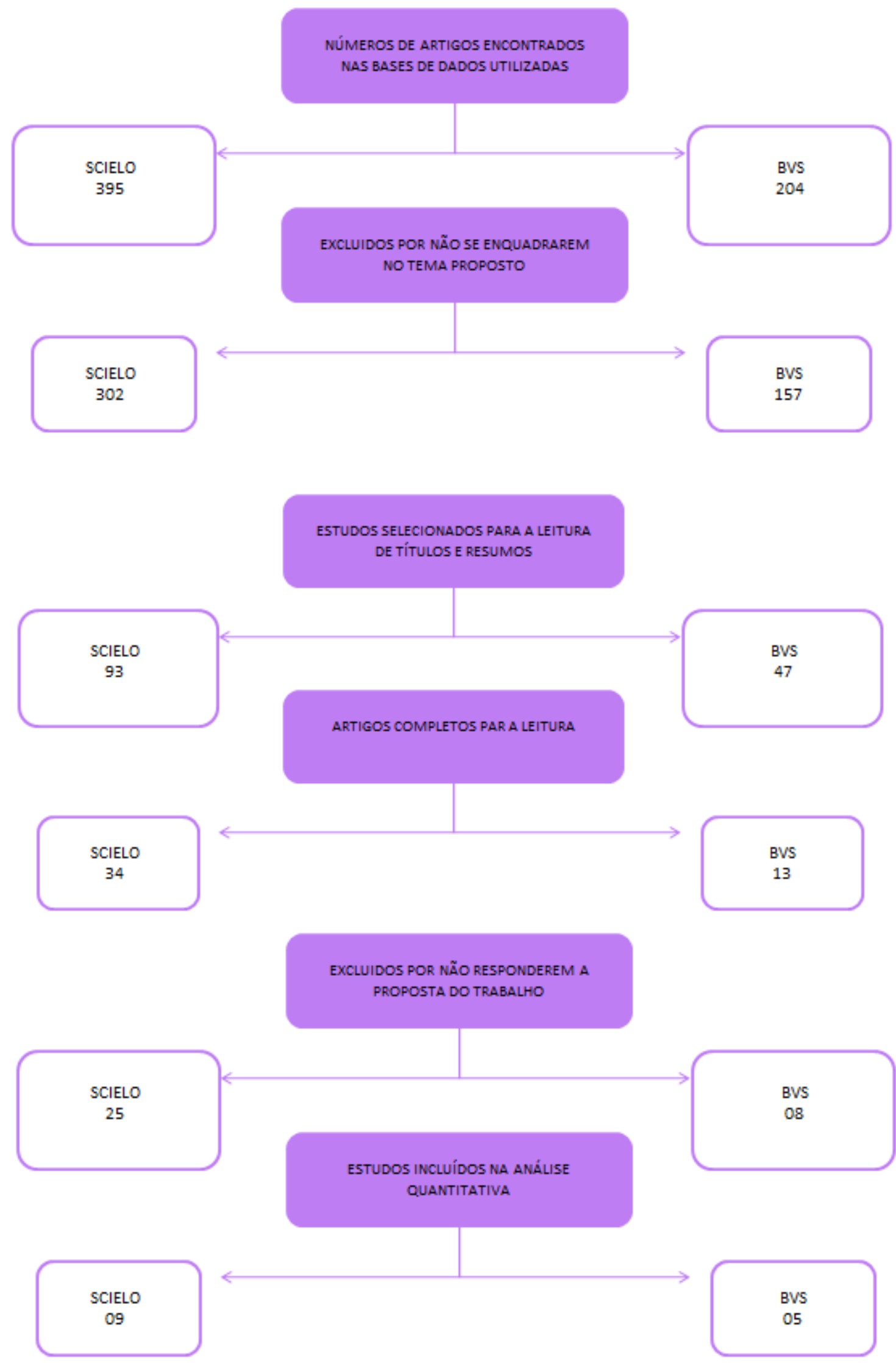

Fonte: Fluxograma elaborado pelos autores. 
Os resultados deste trabalho foram obtidos através da análise quantitativa de publicações sobre o desenvolvimento de trombose em pacientes infectados por SARS-CoV-2 encontrados nas bases de dados Scientific Electronic Library Online e Biblioteca Virtual em Saúde obtendo 395 artigos na Scielo e 204 artigos na BVS. Foram selecionados para a leitura de títulos e resumos o total de 93 e 47 artigos respectivamente e excluídos 302 artigos da Scielo e 157 da BVS por não se enquadrarem no tema proposto. Foi realizada a leitura completa de 34 artigos da Scielo e 13 artigos da BVS, onde 26 da Scielo e 08 da BVS não responderam a proposta do trabalho. Foram incluídos na análise quantitativa 09 artigos da Scielo e 05 artigos da BVS entre os anos de 2019 a 2021.

De acordo com a pesquisa realizada nas bases de dados, foi possivel listar os principais títulos e objetivos dos estudos publicados na literatura, utilizados neste trabalho conforme o Quadro 1.

Quadro 1. Principais títulos e objetivos dos artigos utilizados na pesquisa, Teresina, Brasil, 2021.

\begin{tabular}{|c|c|c|}
\hline $\begin{array}{l}\text { ANO DE } \\
\text { PUBLICAÇÃO }\end{array}$ & TÍTULO & OBJETIVO \\
\hline Roberto et al., 2020. & COVID-19 e eventos tromboembólicos. & $\begin{array}{l}\text { Os autores discutem os principais aspectos dos eventos } \\
\text { tromboembólicos associados ao COVID-19. }\end{array}$ \\
\hline $\begin{array}{l}\text { Franco-López et al., } \\
2020 .\end{array}$ & $\begin{array}{l}\text { Tromboembolismo pulmonar em pacientes com } \\
\text { COVID-19. Angiografia por tomografia } \\
\text { computadorizada:resultados preliminares. }\end{array}$ & $\begin{array}{l}\text { Revisamos retrospectivamente os AngioTCs realizados em } \\
\text { nosso } \\
\text { Radiologia para pacientes com COVID-19 tentando } \\
\text { relacionar os achados radiológicos com os parâmetros } \\
\text { clínicos e os resultados das análises habitualmente } \\
\text { alterados nesta entidade. }\end{array}$ \\
\hline Carvalho et al., 2020. & $\begin{array}{l}\text { Moduladores de coagulação alterados } \\
\text { comprometem os pacientes infectados } \\
\text { com COVID-19. }\end{array}$ & $\begin{array}{c}\text { O objetivo do estudo é compreender a cascata de } \\
\text { sinalização dos diferentes marcadores laboratoriais de } \\
\text { pacientes com COVID-19, a fim de verificar possíveis } \\
\text { distúrbios de coagulação em pacientes gravemente } \\
\text { infectados. }\end{array}$ \\
\hline Tiburi et al., 2021. & $\begin{array}{l}\text { Coagulopatia induzida pelo estado inflamatório } \\
\text { da infecção pela Covid-19. }\end{array}$ & $\begin{array}{c}\text { O presente trabalho teve por objetivo realizar um estudo } \\
\text { de revisão sistemática abordando a correlação entre a } \\
\text { coagulopatia e o contágio pelo SARS-COV-2, enfocando } \\
\text { os principais eventos dessa associação patológica, seu } \\
\text { mecanismo de ação e os biomarcadores atuantes, como } \\
\text { também as estratégias anticoagulantes utilizadas, no } \\
\text { período de } 2020 \text { a } 2021 \text {. }\end{array}$ \\
\hline De Freitas et al., 2021. & Complicações vasculares pós covid-19. & $\begin{array}{l}\text { Como propósito e objetivo geral estudar as complicações } \\
\text { vasculares pós doença, e como objetivos específicos } \\
\text { discorrer sobre a pandemia ocasionada e as consequências } \\
\text { e impactos trazidos pela doença; analisar as complicações } \\
\text { na área da saúde trazidas pela pandemia; discorrer sobre as } \\
\text { complicações vasculares decorrentes do vírus; e por fim } \\
\text { estudar as medidas e abordagens terapêuticas que podem } \\
\text { trazer menos impactos vasculares aos pacientes. }\end{array}$ \\
\hline Brandão et al., 2021. & Papel do Endotélio na COVID-19 Grave. & Compreender o papel do Endotélio na COVID-19 Grave. \\
\hline Rossi, 2020. & $\begin{array}{l}\text { Tromboembolismo venoso em pacientes } \\
\text { COVID-19. }\end{array}$ & $\begin{array}{c}\text { Neste estudo, revisamos os principais estudos e evidências } \\
\text { disponíveis até o momento sobre o diagnóstico, profilaxia } \\
\text { e recomendações de tratamento do TEV em pacientes } \\
\text { COVID-19. }\end{array}$ \\
\hline Passos et al., 2020. & $\begin{array}{c}\text { Infecção pelo SARS-Cov-2 e } \\
\text { Tromboembolismo Pulmonar - Comportamento } \\
\text { Pró - Trombótico da COVID-19. }\end{array}$ & $\begin{array}{l}\text { Descrição de caso de paciente diagnosticado com infecção } \\
\text { pelo SARS-CoV-2 evoluindo com tromboembolismo } \\
\text { pulmonar sem evidência de trombose periférica. }\end{array}$ \\
\hline Brandão et al., 2020. & $\begin{array}{l}\text { COVID-19 grave: entenda o papel da } \\
\text { imunidade, do endotélio e da coagulação na } \\
\text { prática clínica. }\end{array}$ & $\begin{array}{c}\text { O objetivo é provocar uma maior reflexão sobre a } \\
\text { influência desses fatores na abordagem terapêutica dessa } \\
\text { nova doença. }\end{array}$ \\
\hline Casella, 2020. & $\begin{array}{c}\text { Fisiopatologia da trombose associada à infecção } \\
\text { pelo SARS-CoV-2. }\end{array}$ & $\begin{array}{l}\text { Descrever a fisiopatologia da trombose associada à } \\
\text { infecção pelo SARS-CoV- } 2 \text {. }\end{array}$ \\
\hline
\end{tabular}




\begin{tabular}{|c|c|c|}
\hline Soeiro et al., 2020. & $\begin{array}{c}\text { Posicionamento sobre Uso de Antiplaquetários e } \\
\text { Anticoagulantes nos Pacientes Infectados pelo } \\
\text { Novo Coronavírus (COVID-19)-2020. }\end{array}$ & $\begin{array}{c}\text { Posicionamento sobre uso de antiplaquetários e } \\
\text { anticoagulantes nos pacientes infectados pelo novo } \\
\text { Coronavírus. }\end{array}$ \\
\hline $\begin{array}{c}\text { Nascimento } \text { et al., } \\
2020 .\end{array}$ & $\begin{array}{c}\text { COVID-19 e Estado de Hipercoagulabilidade: } \\
\text { Uma Nova Perspectiva Terapêutica. }\end{array}$ & $\begin{array}{c}\text { Uma nova perspectiva terapêutica a respeito da COVID-19 } \\
\text { e estado de hipercoagulabilidade. }\end{array}$ \\
\hline $\begin{array}{c}\text { De Borba Batschauer; } \\
\text { Jovita, 2020. }\end{array}$ & $\begin{array}{c}\text { Hemostasia e COVID-19: fisiopatologia, } \\
\text { exames laboratoriais e terapia anticoagulante. }\end{array}$ & $\begin{array}{c}\text { Compreender a relação entre os mecanismos hemostáticos } \\
\text { e a infecção pela COVID-19 torna-se de fundamental } \\
\text { importância para auxiliar os profissionais de saúde nas } \\
\text { condutas laboratoriais, clínicas e terapêuticas no } \\
\text { monitoramento dos casos e melhores encaminhamentos. }\end{array}$ \\
\hline Cunha et al., 2021. & $\begin{array}{c}\text { Incidência, diagnóstico, métodos de tratamento e } \\
\text { resultados de doença tromboembólica venosa } \\
\text { clinicamente suspeita em pacientes com } \\
\text { COVID-19 em um hospital quaternário no } \\
\text { Brasil. }\end{array}$ & $\begin{array}{c}\text { Este estudo apresenta uma análise epidemiológica dos } \\
\text { episódios manifestos de TEV em um grupo de pacientes } \\
\text { internados por COVID-19. }\end{array}$ \\
\hline
\end{tabular}

Fonte: SCIELO e BVS.

Desta forma, foi possível listar os principais resultados e conclusões dos estudos publicados na literatura, conforme o

Quadro 2.

Quadro 2. Resultados e conclusões dos artigos utilizados, Teresina, Brasil, 2021.

\begin{tabular}{|c|c|c|}
\hline PUBLICAÇÃO & RESULTADOS & CONCLUSÕES \\
\hline Roberto et al., 2020. & $\begin{array}{l}\text { A orientação provisória da OMS recomenda } \\
\text { doses profiláticas diárias de heparinas de baixo peso } \\
\text { molecular (HBPM) ou duas vezes ao dia de heparina não } \\
\text { fracionada subcutânea. E, se profilaxia farmacológica é } \\
\text { contraindicada, a indicação alternativa é a profilaxia } \\
\text { mecânica intermitente (compressão pneumática) que deve } \\
\text { ser considerada em pacientes imobilizados. }\end{array}$ & $\begin{array}{l}\text { Recomenda-se tromboprofilaxia de rotina de todos } \\
\text { os pacientes hospitalizados com COVID-19, } \\
\text { independentemente do escore de risco, sendo esta } \\
\text { farmacológica ou mecânica. O uso de } \\
\text { Anticoagulantes orais e novos tratamentos } \\
\text { hemostáticos oferecem grandes vantagens que são } \\
\text { ainda mais óbvias em tempos de crise. }\end{array}$ \\
\hline $\begin{array}{c}\text { Franco-López et al., } \\
2020 .\end{array}$ & $\begin{array}{l}\text { Os pacientes apresentavam um D-dímero médio na } \\
\text { admissão de } 1.620,5 \text {, que aumentou em } 17 \text { dos } 18 \text { pacientes } \\
\text { durante a admissão, permanecendo elevados no paciente } \\
\text { restante. eu sei observa que em um de nossos centros o D- } \\
\text { Dimer foi calculado até um máximo de } \\
4.400 \text { e não foi quantificado acima deste valor. O pró-BNP } \\
\text { foi registrado em } 13 \text { dos } 18 \text { pacientes com um valor médio } \\
\text { de } 1.066,69 \text { (intervalo } 86-3220 \text { ). }\end{array}$ & $\begin{array}{l}\text { Em conclusão, queremos chamar a atenção para a } \\
\text { possibilidade de que uma complicação } \\
\text { Relativamente comum de infecção por COVID-19 } \\
\text { é PTSD. Em pacientes com hipóxia e Valores } \\
\text { elevados de dímero-D com tendência à } \\
\text { normalização da ferritina são importantes descartar } \\
\text { ou confirmar a presença de EP devido às } \\
\text { implicações terapêuticas que tem para o paciente. }\end{array}$ \\
\hline $\begin{array}{l}\text { Carvalho et al., } \\
2020 .\end{array}$ & $\begin{array}{l}\text { Os leucócitos ativados podem danificar o endotélio capilar } \\
\text { e interromper o estado tromboprotetor das células } \\
\text { endoteliais (SMADJA et al., 2020) resultando na liberação } \\
\text { de ativadores de plasminogênio, o que pode explicar as } \\
\text { altas concentrações de D-dímero como produto de } \\
\text { degradação de fibrina, na tentativa do organismo de } \\
\text { pacientes graves com COVID-19 desfazer coágulos (LEVI } \\
\text { et al., 2020). }\end{array}$ & $\begin{array}{l}\text { As principais alterações evidenciadas no estudo } \\
\text { foram elevação do D-dímero e do fibrinogênio, } \\
\text { bem como prolongamento do tempo de } \\
\text { protrombina e do tempo de tromboplastina parcial } \\
\text { ativada. Todos esses marcadores estão } \\
\text { relacionados a um pior prognóstico da COVID-19, } \\
\text { visto que são mais evidentes em pacientes em } \\
\text { estado grave da doença. }\end{array}$ \\
\hline Tiburi et al., 2021. & $\begin{array}{l}\text { A pneumonia causada pelo Sars-Cov-2 pode apresentar } \\
\text { diversas complicações, desde manifestações pulmonares } \\
\text { graves como SDRA (síndrome da angústia respiratória } \\
\text { grave) e TEP até coagulopatias, sendo essa última mais } \\
\text { associado a prognóstico reservado (HELMS et al., 2020). } \\
\text { Nesse contexto, casos de maior gravidade e } \\
\text { morbimortalidade apresentavam fatores epidemiológicos } \\
\text { comuns para o desenvolvimento de desfechos } \\
\text { tromboembólicos, como a presença de comorbidades em } \\
\text { 60,6\% dos pacientes com COVID-19 grave (TANG et al., } \\
\text { 2020). }\end{array}$ & $\begin{array}{l}\text { Dessa forma, em vista dos impactos negativos da } \\
\text { coagulopatia associada à infecção, é de suma } \\
\text { importância um diagnóstico precoce e oferta de } \\
\text { suporte terapêutico eficaz, identificando fatores de } \\
\text { risco para o desenvolvimento da condição e } \\
\text { instituindo medidas profiláticas ideais para evitar o } \\
\text { curso natural da complicação. }\end{array}$ \\
\hline $\begin{array}{c}\text { De Freitas et al. } \\
2021 .\end{array}$ & $\begin{array}{l}\text { Com relação ainda a fisiopatologia, é importante ressaltar } \\
\text { que existem aspectos relevantes a serem realçados, como o } \\
\text { fato de as explicações cientificas ainda estarem no campo } \\
\text { das hipóteses; os processos patológicos observados são }\end{array}$ & $\begin{array}{l}\text { Conforme explicitado no curso do estudo, } \\
\text { processos inflamatórios no organismo de pacientes } \\
\text { infectados pelo coronavírus podem levar a quadros } \\
\text { de complicações vasculares como a trombose, e }\end{array}$ \\
\hline
\end{tabular}




\begin{tabular}{|c|c|c|}
\hline & $\begin{array}{c}\text { predominantemente concomitantes e se estimulam } \\
\text { mutuamente; dificuldades de quantificação dos impactos de } \\
\text { eventos que ocorrem em escala molecular e celular nos } \\
\text { desfechos clínicos finais das complicações vasculares como } \\
\text { o evento trombótico. }\end{array}$ & $\begin{array}{l}\text { neste sentido, pessoas com pré-disposição a tais } \\
\text { complicações devem receber acompanhamento de } \\
\text { especialistas como angiologista ou cirurgião } \\
\text { vascular, devendo manter a supervisão de } \\
\text { especialistas mesmo após a sua recuperação. }\end{array}$ \\
\hline $\begin{array}{l}\text { Brandão et al., } \\
2021 .\end{array}$ & $\begin{array}{l}\text { Na fase hiperinflamatória da COVID-19, as medicações } \\
\text { que inibam ou reduzam os efeitos das citocinas } \\
\text { próinflamatórias são muito pertinentes e devem ser levadas } \\
\text { em consideração. Os inibidores de IL-6, assim como os } \\
\text { glicocorticoides, poderiam evitar ou amenizar a tempestade } \\
\text { de citocinas.23 Novas medicações moduladoras da resposta } \\
\text { inflamatória são fundamentais nessa fase para evitar a } \\
\text { inflamação excessiva, que agride intensamente o endotélio } \\
\text { e os diversos órgãos, podendo culminar com falência de } \\
\text { múltiplos órgãos até a morte. }\end{array}$ & $\begin{array}{c}\text { Em resumo, ressalta-se que a função endotelial é } \\
\text { fator fundamental na progressão dos estágios } \\
\text { clínicos da COVID-19, pois a disfunção crônica do } \\
\text { endotélio, que acontece nas doenças pré-existentes, } \\
\text { favorece diretamente a evolução para a forma } \\
\text { grave da doença. }\end{array}$ \\
\hline Rossi, 2020. & $\begin{array}{l}\text { Nos pacientes que apresentam DD elevado ou suspeita } \\
\text { clínica elevada de TEP e quando houver dificuldade de } \\
\text { acesso a tomografia, tem sido recomendada a realização de } \\
\text { exames complementares à beira do leito. O ecocardiograma } \\
\text { transtorácico é capaz de identificar sinais de sobrecarga de } \\
\text { ventrículo direito, que podem sugerir a presença de TEP. O } \\
\text { EDV, realizado pela técnica point-of-care, pode verificar a } \\
\text { presença de trombose venosa profunda no eixo fêmoro- } \\
\text { poplíteo. }\end{array}$ & $\begin{array}{c}\text { O uso de HBPM em doses profiláticas e } \\
\text { terapêuticas vem demonstrando benefício clínico e } \\
\text { baixo risco de complicações hemorrágicas. Dessa } \\
\text { forma, concluo que existe elevado risco de TEV na } \\
\text { pandemia COVID-19 e que a profilaxia e o } \\
\text { tratamento com o uso de heparina em suas diversas } \\
\text { formas posológicas devem ser realizados de forma } \\
\text { agressiva nos pacientes que não apresentem risco } \\
\text { elevado de complicações hemorrágicas. }\end{array}$ \\
\hline Passos et al., 2020. & $\begin{array}{l}\text { As complicações cardiovasculares têm sido apresentadas de } \\
\text { forma expressiva e variável na vigência da infecção pelo } \\
\text { novo coronavirus.5 Na apresentação mais grave da } \\
\text { COVID-19, observam-se altos níveis de dímero-D, que tem } \\
\text { sido associado a aumento da mortalidade. }\end{array}$ & $\begin{array}{c}\text { A infecção pelo SARS-Cov-2 se apresenta com um } \\
\text { fenótipo variável, sendo frequentes os relatos de } \\
\text { complicações cardiovasculares e a presença de um } \\
\text { estado pró-trombótico, por mecanismos ainda não } \\
\text { totalmente elucidados. }\end{array}$ \\
\hline $\begin{array}{c}\text { Brandão et al., } \\
2020 .\end{array}$ & $\begin{array}{l}\text { Diante de uma pandemia, as condutas vão sendo } \\
\text { readequadas de acordo com a evolução do conhecimento e } \\
\text { muito do que já é rotina no tratamento de pacientes graves } \\
\text { está sendo aplicado no tratamento da COVID-19. As } \\
\text { principais complicações da COVID-19 são SARS, SIC, } \\
\text { TEV e CIVD. Todas elas são bem conhecidas na sepse e na } \\
\text { COVID-19 parecem ser consequência da resposta } \\
\text { inflamatória desregulada. }\end{array}$ & $\begin{array}{c}\text { Em resumo, enquanto aguardamos a vacina, talvez } \\
\text { o melhor tratamento para a COVID-19 seja aquele } \\
\text { que englobe terapias que melhoram as condições } \\
\text { cardiovasculares e metabólicas dos pacientes, além } \\
\text { de medicações que reduzam a replicação viral, a } \\
\text { hiperinflamação e o risco de trombose. }\end{array}$ \\
\hline Casella, 2020. & $\begin{array}{l}\text { A marcante elevação do dímero D foi observada em } \\
\text { pacientes Covid+, sendo superlativa nos pacientes mais } \\
\text { graves e claramente associada a piores prognósticos14. } \\
\text { Embora não totalmente compreendida, pode ser explicada } \\
\text { pela ocorrência de maciça trombose microvascular e pela } \\
\text { ativação do sistema fibrinolítico por estímulo viral direto } \\
\text { com posterior inibição deste. }\end{array}$ & $\begin{array}{c}\text { A compreensão dos processos fisiopatológicos que } \\
\text { resultam em tais fenômenos trombóticos ainda é } \\
\text { incompleta. Também, a relação de causa e efeito } \\
\text { entre eventos trombóticos e a gravidade da } \\
\text { infecção pelo SARS-CoV-2 ainda precisa ser mais } \\
\text { bem compreendida. }\end{array}$ \\
\hline Soeiro et al., 2020. & $\begin{array}{l}\text { A terapia anticoagulante em pacientes com COVID-19 } \\
\text { grave e indícios de SIC e/ou com D-dímero muito elevado } \\
\text { em associação a outros biomarcadores que denotam } \\
\text { gravidade, na ausência de contraindicação à } \\
\text { anticoagulação, pode ser considerada uma estratégia } \\
\text { terapêutica fundamentada no consenso de especialistas e } \\
\text { em poucos estudos retrospectivos. Adicionalmente, essa } \\
\text { estratégia requer a utilização de protocolos institucionais } \\
\text { rígidos que permitam a vigilância e a rápida. intervenção } \\
\text { frente a complicações.2 }\end{array}$ & $\begin{array}{l}\text { As evidências a respeito da COVID-19 e suas } \\
\text { interações com os sistemas de coagulação e } \\
\text { ativação plaquetária ainda são iniciais. Existem } \\
\text { fortes indícios de que essa via possa ser um alvo } \\
\text { terapêutico importante. }\end{array}$ \\
\hline $\begin{array}{c}\text { Nascimento et al., } \\
2020 .\end{array}$ & $\begin{array}{l}\text { De acordo com o exposto, é razoável alinhar a } \\
\text { fisiopatologia pró-trombótica já descrita na sepse com } \\
\text { aspectos intrínsecos do novo coronavírus e, portanto, } \\
\text { analisar individualmente o potencial benefício do uso de } \\
\text { anticoagulantes em grupos selecionados de pacientes. Um } \\
\text { estudo retrospectivo realizado no hospital de Tongji } \\
\text { (Wuhan, China) descreveu a ocorrência de menor taxa de } \\
\text { mortalidade nos pacientes com COVID-19 grave que } \\
\text { fizeram uso de anticoagulante, heparina não fracionada ou } \\
\text { heparina de baixo peso molecular (HBPM), e apresentavam }\end{array}$ & $\begin{array}{l}\text { Em conclusão, a fisiopatologia da COVID-19 } \\
\text { envolve ativação da resposta inflamatória e } \\
\text { indução do sistema trombótico. No momento, } \\
\text { consenso de especialistas sugere tratamento com } \\
\text { anticoagulante para pacientes que tenham fenótipo } \\
\text { prócoagulante (dímero-D elevado, prolongamento } \\
\text { de tempo de protrombina e aumento dos níveis } \\
\text { plasmáticos de fragmentos da fibrina). Mais } \\
\text { estudos são necessários para confirmar o real papel } \\
\text { da anticoagulação na prevenção de complicações }\end{array}$ \\
\hline
\end{tabular}




\begin{tabular}{|c|c|c|}
\hline & $\begin{array}{c}\text { escore SIC } \geq 4 \text { e/ou dímero-D muito elevado (> } 6 \text { vezes o } \\
\text { limite superior da normalidade). }\end{array}$ & da COVID-19. \\
\hline $\begin{array}{c}\text { De Borba } \\
\text { Batschauer; Jovita, } \\
2020 .\end{array}$ & $\begin{array}{l}\text { A coagulopatia na COVID-19 é um evento frequentemente } \\
\text { descrito na literatura recente e está associada a um estado } \\
\text { de hipercoagulabilidade; entretanto, os processos } \\
\text { moleculares associados à sua causa ainda permanecem } \\
\text { desconhecidos e necessitam de maior investigação.(6) O } \\
\text { mecanismo de coagulação é um processo que envolve a } \\
\text { interação de três componentes-chave: células endoteliais, } \\
\text { plaquetas e fatores de coagulação. }\end{array}$ & $\begin{array}{l}\text { Conclui-se que, embora os mecanismos específicos } \\
\text { ainda não estejam claros, a infecção pelo SARS- } \\
\text { CoV-2 obviamente envolve processos } \\
\text { potencialmente deletérios na coagulação e } \\
\text { inflamação.(20) Pacientes com COVID19 } \\
\text { apresentam um quadro de hipercoagulabilidade, } \\
\text { associada à infecção severa, evoluindo para EP } \\
\text { e/ou TVP, e alguns casos mais graves de CIVD. }\end{array}$ \\
\hline Cunha et al., 2021. & $\begin{array}{c}\text { Durante o período do estudo, foram admitidos } 484 \text { casos } \\
\text { confirmados de COVID-19, } 64 \text { dos quais apresentavam } \\
\text { sintomas de TEV e } 13 \text { dos quais tinham TEV sintomático } \\
\text { confirmado (2,68\% da amostra total e } 20,31 \% \text { dos casos } \\
\text { sintomáticos). A maioria dos casos ( } 76,92 \%) \text { ocorreu em } \\
\text { terapia intensiva. }\end{array}$ & $\begin{array}{l}\text { A prevalência de TEV em pacientes hospitalizados } \\
\text { com COVID-19 foi de } 2,7 \% \text { e maior em unidades } \\
\text { de terapia intensiva. A instituição precoce da } \\
\text { profilaxia e anticoagulação total imediata quando o } \\
\text { TEV é diagnosticado devem ser os objetivos } \\
\text { daqueles que tratam esse tipo de paciente. }\end{array}$ \\
\hline
\end{tabular}

Fonte: SCIELO e BVS.

Baseando-se nos resultados e conclusões dos artigos utilizados, pode-se observar que o vírus que causa COVID-19, a doença que continua matando milhares de pessoas em todo o mundo, afeta principalmente os pulmões de forma aguda e severa. Este patógeno é descrito como um beta coronavírus com material genético complexo com RNA de fita simples e estudos recentes mostram que pessoas infectadas com esta doença, além da complicação no desempenho respiratório, eles também sofrem de colapso de vários órgãos e trombose, como lesão miocárdica aguda causada por isquemia e acidente vascular cerebral isquêmico (Tiburi et al., 2021).

O Quadro 3 demonstra os principais fatores de risco na infecção pelo vírus responsável pela COVID-19.

Quadro 3. Apresenta os principais fatores de risco na infecção por SARS-CoV-2.

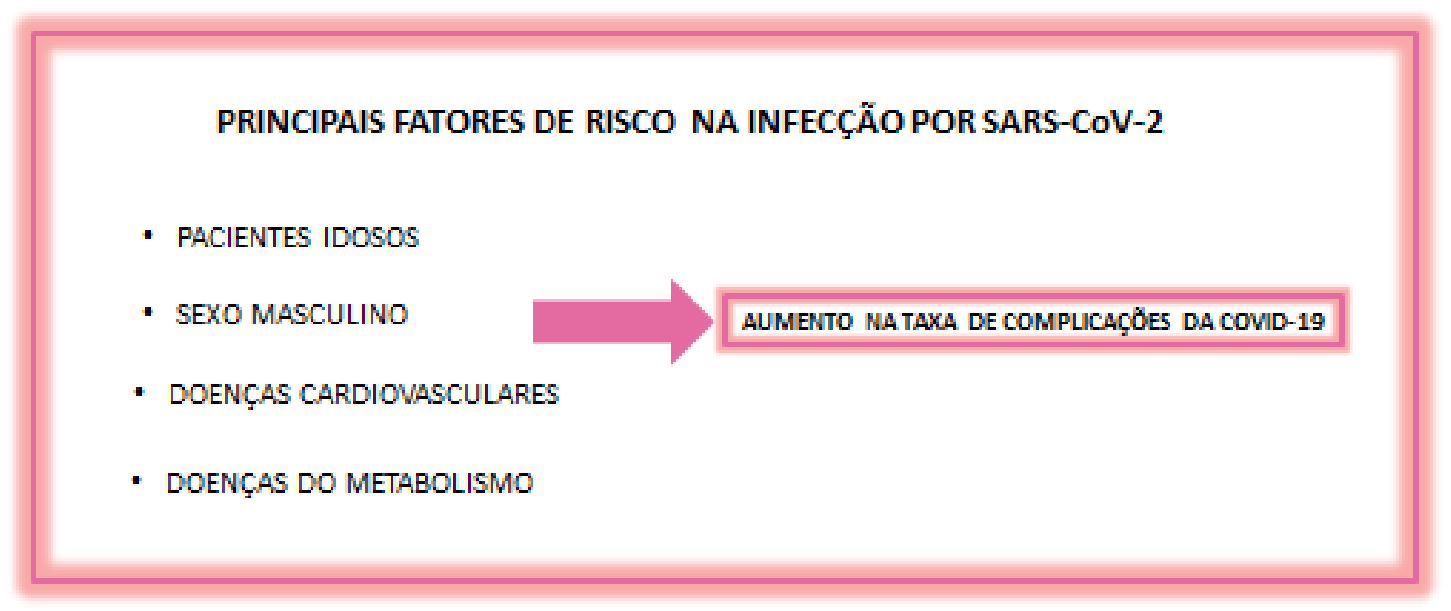

Fonte: Adaptado de Brandão (2020).

Pacientes idosos, do sexo masculino, com doenças cardiovasculares e/ou metabólicas apresentam uma chance maior de evolução desfavorável da COVID-19. “Tempestades” de citocinas, proteínas de fase aguda elevadas e biomarcadores de lesão cardíaca são características da forma grave e preditivos de óbito (Brandão et al., 2020).

Alguns estudos sugerem que pacientes com COVID-19 podem apresentar um estado de hipercoagulabilidade, em que ocorre um aumento dos níveis séricos de fator VIII, fator de Von Willebrand e fibrinogênio. A hipercoagulabilidade pode ter influência no desenvolvimento de trombose venosa profunda dos membros inferiores e/ou embolia pulmonar nesses pacientes. A causa desse aumento de coagulabilidade ainda permanece desconhecida, toda via, acredita-se que ela ocorra devido ao aumento 
dos fatores pró-coagulantes e de uma possível redução dos fatores anticoagulantes presentes no plasma dos pacientes infectados (Roberto et al., 2020).

O tromboembolismo venoso é considerado um grave problema de saúde que afeta 1 em cada 1.000 adultos em todo o mundo anualmente. Estudos epidemiológicos têm mostrado que antes de medidas preventivas hospitalares consistentes, cerca de $55 \%$ a $60 \%$ de todos os casos de TEV estavam relacionados à hospitalização e são diagnosticados durante a hospitalização ou nos primeiros 90 dias após a alta do paciente (Cunha et al., 2021).

Os pacientes com infecção por SARS-CoV-2 desenvolvem uma significativa inflamação, em consequência de uma tempestade de citocinas. A coagulopatia associada à COVID-19 (CAC) está sendo utilizada atualmente para descrever os distúrbios na coagulação. Entretanto, os mecanismos pró-coagulantes ativados pelo SARS-CoV-2 são provenientes de uma resposta inflamatória profunda, e não de um processo intrínseco ao vírus (Carvalho et al., 2020).

A trombose microvascular afeta principalmente os pulmões, até $30 \%$ causa embolia pulmonar Nos casos mais graves, além de complicações renais e cardíacas, podem provocar falência múltipla de órgãos (De Borba Batschauer; Jovita, 2020).

O endotélio é um órgão ativo, que possui funções endócrinas, parácrinas e autócrinas, necessárias para a regulação do tônus e a manutenção da homeostase vascular. Na COVID-19, devido à agressão direta do vírus ao endotélio, é recrutada células do sistema imune, podendo resultar em disfunção endotelial generalizada associada a apoptose (Brandão et al., 2020).

O endotélio vascular em condições fisiológicas possui uma variedade de mecanismos para inibir eventos trombóticos. Em contrapartida, estímulos patológicos no nível celular ou molecular irão estimular a trombose endotelial, como aumento da expressão de TF e PAI-1, ativação plaquetária, liberação de citocinas inflamatórias e expressão de anticoagulantes endógenos (como a trombomodulina) (Casella, 2020).

Pacientes com o tipo grave de coronavírus apresentam anormalidades hemostáticas semelhantes à coagulopatia intravascular disseminada e sepse. A diferença é que a presença do coronavírus aumenta a chance do estado de hipercoagulabilidade. Eventos persistentes relacionados à trombose são comuns em pacientes em estado crítico. Estudos mostram que a atividade dos anticoagulantes naturais é baixa, e os valores de fibrinogênio (FIB), tempo de protrombina (PT), tempo de tromboplastina parcial ativada (APTT) e dímero D estão significativamente aumentados, o que pode indicar coagulopatia associada à SARS-CoV-2, uma doença que afeta o endotélio e que leva ao aumento da ativação plaquetária e do aumento do processo de coagulação, levando a manifestações clínicas pré-trombóticas associadas à COVID-19 (Tiburi et al., 2021).

O desequilíbrio da coagulação em pacientes com COVID-19 pode ser explicado por diversos mecanismos, como por exemplo as armadilhas externas de neutrófilos, liberadas a partir de sua ativação, que constitui uma mistura de DNA, histonas e nucleossomos, podendo agregar substâncias pró-coagulantes relacionadas ao estado hipercoagulável do plasma e aumento do risco de trombose. Outro mecanismo é a inflamação causada pelo o vírus, isso também está intimamente relacionado à trombose, porque as citocinas pró-inflamatórias são reguladoras da ativação da coagulação, o que pode explicar a coagulação relacionada à COVID-19 (CAC) capaz de aumentar a produção de trombina e reduzir a fibrinólise endógena. Essa resposta inflamatória inadequada é a causa da progressão do CAC, e o aumento do dímero D pode estar relacionado a um pior prognóstico da doença (Carvalho et al., 2020).

Estudos demonstraram que os pacientes com Covid-19 (especialmente quando pertencentes a grupos com comorbidades) podem ter um alto risco de eventos tromboembólicos, como consequências da doença. Os eventos tromboembólicos mais comuns são tromboembolismo venoso, também conhecido como TEV. O risco de que o paciente desenvolva problemas vasculares quando infectados pelo vírus da COVID-19 é alto. Um dos tratamentos recomendados para o caso de TEV é o uso de anticoagulantes, entretanto, ainda faltam pesquisas sobre a relação risco-benefício da adoção de estratégias anticoagulantes, inclusive a longo prazo (De Freitas et al., 2021).

Diversos estudos relatam eventos trombóticos em veias (trombose venosa profunda, embolia pulmonar) e artérias 
(acidente vascular cerebral isquêmico, trombose arterial periférica) em pacientes infectados com SARSCoV-2. Os métodos desses relatórios variam amplamente, o que dificulta a disponibilidade de dados homogêneos. Em contraste, foi observado que pacientes gravemente enfermos infectados com SARS-CoV-2 tiveram uma incidência aumentada de eventos tromboembólicos venosos, comparados a pacientes com infecções respiratórias graves causadas por outros patógenos (Casella, 2020).

As principais alterações da coagulação na COVID-19 são: aumento do dímero D, tempo de fibrinogênio e protrombina e diminuição da fibrinólise. A contagem de plaquetas pode diminuir em estágios onde a doença está mais avançada e é um preditor de mortalidade. O risco aumentado de trombose também ocorre nas artérias, podendo ocorrer diferentes manifestações clínicas, como acidente vascular cerebral, isquemia mesentérica, infarto agudo do miocárdio e oclusão arterial de membros inferiores, dependendo do leito arterial envolvido (Brandão et al., 2021).

O aumento do dímero D na forma grave de COVID-19 e a sobreposição de sintomas respiratórios da doença de base às do tromboembolismo pulmonar dificultam o diagnóstico precoce deste último. Atenção especial deve ser dada às seguintes alterações: hipoxemia refratária, alterações do ECG, taquicardia sinusal que não pode ser explicada pelo quadro clínico atual e disfunção ventricular direita, sendo utilizadas no diagnóstico de trombose pulmonar e no início da terapia anticoagulante apropriada (Passos et al., 2020).

A presença de hipóxia e o aumento do D-dímero são dados que fazem com que haja uma sobreposição entre COVID-19 e tromboembolismo pulmonar. Pode haver vários fatores que afetam o desenvolvimento do trombo, a partir de fatores inflamatórios locais para fixação e estados pró-coagulantes. A presença de trombo na árvore vascular pulmonar é uma indicação para terapia anticoagulante nesses pacientes (Franco-López et al., 2020).

$\mathrm{O}$ dímero $\mathrm{D}$ está associado a uma taxa de mortalidade mais elevada e parece aumentar gradualmente à medida que a infecção piora. Estágios do desenvolvimento e exacerbação da síndrome da angústia respiratória aguda (SDRA) e a piora do padrão radiológico são marcadas pelo aumento expressivo de D-dímero, observando-se nos piores casos de injúria miocárdica e coagulação intravascular disseminada (CIVD) (Soeiro et al., 2020).

O dímero-D é um produto da degradação da fibrina e, quando aumenta, está associado a uma mortalidade mais elevada. Com base na experiência clínica e na análise de alguns estudos descritivos é enfatizado o papel da hipercoagulabilidade na fisiopatologia da COVID-19, porque o nível de dímero D varia com o agravamento da infecção. No estágio da doença em que a SDRA se desenvolve e o padrão radiológico se deteriora, o dímero-D aumenta significativamente e, nos casos mais graves, são observados danos ao miocárdio e CIVD (Nascimento et al., 2020).

Uma incidência relativamente alta de trombose e doenças tromboembólicas foi observada em pacientes com COVID-19, o que pode ser devido à influência direta do SARSCoV-2 ou ao mecanismo indireto da própria infecção. Além disso, as interações medicamentosas entre as terapias para COVID-19 e os antiagregantes plaquetários ou anticoagulantes, bem como a suspensão inadvertida dos anticoagulantes também podem levar ao estado pró-trombótico encontrado na doença (Passos et al., 2020).

No tromboembolismo venoso, os pacientes que estão hospitalizados devem receber tromboprofilaxia farmacológica com heparina de baixo peso molecular ou fondaparinux (de preferência heparina não fracionada), a menos que o risco de sangramento exceda o risco de trombose, quando então é realizada a profilaxia mecânica. $\mathrm{O}$ ajuste da dose da heparina é feito de acordo com o índice de massa corpórea e com o clearance de creatinina. A indicação da heparinização plena é feita em casos de forte suspeita clínica ou em casos já confirmados de tromboembolismo venoso (Brandão et al., 2021).

Pacientes em uso de medicamentos antitrombóticos devem ser orientados a continuar usando o medicamento, mas devem ser alertados sobre o risco de interações medicamentosas entre esses medicamentos e os medicamentos prescritos na COVID-19, especialmente antivirais hormonais e não hormonais e anti-inflamatórios. Geralmente não há indicações de medicamentos para prevenir TEV; no entanto a profilaxia com heparina de baixo peso molecular (HBPM) pode ser considerada, especialmente nos 
portadores de risco aumentado para TEV desde que não haja alto risco para sangramento (Rossi, 2020).

\section{Conclusão}

A infecção pelo SARS-Cov-2 se apresenta com uma variedade de complicações, sendo frequentes os relatos de problemas cardiovasculares e a presença de um estado pró-trombótico, causado por mecanismos ainda não totalmente elucidados. As evidências sobre COVID-19 e sua interação com o sistema de coagulação e ativação plaquetária ainda não estão totalmente esclarecidos. Há fortes evidências de que essa abordagem pode ser um importante alvo terapêutico. No entanto, pesquisas mais robustas ainda são necessárias para determinar a verdadeira importância do mecanismo pró-trombótico e a melhor terapia a ser utilizada neste grupo de pacientes.

Em vista dos efeitos negativos da coagulopatia relacionada à infecção, são de extrema importância o diagnóstico precoce e suporte de tratamento eficaz, identificando os fatores de risco para progressão da doença e desenvolvimento de medidas preventivas ideais para evitar complicações. De acordo com estudos científicos a fisiopatologia da COVID-19 envolve a ativação da resposta inflamatória e a indução do sistema trombótico. Quando o tromboembolismo é diagnosticado, a prevenção precoce e a anticoagulação imediata e abrangente devem ser os principais objetivos para o tratamento desses pacientes.

Atualmente, o consenso de especialistas recomenda terapia anticoagulante para pacientes com aumento dos níveis de dímero D, tempo de protrombina prolongado e níveis elevados de fragmento de fibrina no plasma. É essencial que mais pesquisas sejam realizadas para confirmar o verdadeiro papel da anticoagulação na prevenção das complicações da COVID-19.

\section{Referências}

Brandão, S. C. S., Godoi, E. T. A. M., Ramos, J. D. O. X., Melo, L. M. M. P. D., Dompieri, L. T., Brindeiro, D. F., \& Sarinho, E. S. C. (2021). Papel do Endotélio na COVID-19 Grave. Arquivos Brasileiros de Cardiologia, 115, 1184-1189.

Brandão, S. C. S., Godoi, E. T. A. M., Ramos, J. D. O. X., Melo, L. M. M. P. D., \& Sarinho, E. S. C. (2020). COVID-19 grave: entenda o papel da imunidade, do endotélio e da coagulação na prática clínica. Jornal Vascular Brasileiro, 19.

Carvalho, A. C. S., de Albuquerque Barros, L. S., Tenório, E. C. P. T., Lopes, T. P., Lopes, L. P., \& da Cruz, C. M. (2020). Moduladores de coagulação alterados comprometem os pacientes infectados com COVID-19. Brazilian Journal of Health Review, 3(5).

Casella, I. B. (2020). Fisiopatologia da trombose associada à infecção pelo SARS-CoV-2. Jornal Vascular Brasileiro, $1677-7301$.

Cunha, M. J. S., Pinto, C. A. V., Guerra, J. C. D. C., Tachibana, A., Portugal, M. F. C., Ferraz, L. J. R., \& Wolosker, N. (2021). Incidence, diagnosis, treatment methods, and outcomes of clinically suspected venous thromboembolic disease in patients with COVID-19 in a quaternary hospital in Brazil. Jornal Vascular Brasileiro, 20.

de Borba Batschauer, A. P., \& JOVITA, H. W. (2020). Hemostasia e COVID-19: fisiopatologia, exames laboratoriais e terapia anticoagulante. A Tempestade do Coronavírus, 52(2), 138-42.

De Freitas, A. L. G. C., Santos, M. S., \& Dos Santos, J. A. B. (2021). Complicações vasculares pós covid-19. Brazilian Journal of Health Review, 4(3), 1309013105 .

Franco-López, Á., Escribano Poveda, J., \& Vicente Gilabert, N. (2020). Tromboembolismo Pulmonar en los pacientes con COVID-19. Angiografía con tomografía computarizada: resultados preliminares. Journal of Negative and No Positive Results, 5(6), 616-630.

Nascimento, J. H. P., Gomes, B. F. D. O., Carmo, P. R. D., Petriz, J. L. F., Rizk, S. I., Costa, I. B. S. D. S., \& Oliveira, G. M. M. D. (2020). COVID-19 e estado de hipercoagulabilidade: uma nova perspectiva terapêutica. Arquivos Brasileiros de Cardiologia, 114, 829-833.

Passos, H. D., Alves, M. C., Baumworcel, L., Vieira, J. P. C., Garcez, J. D. S., \& Sousa, A. C. S. (2020). Infecção pelo SARS-Cov-2 e Tromboembolismo Pulmonar-Comportamento Pró-Trombótico da COVID-19. Arquivos Brasileiros de Cardiologia, 115, 142-145.

Roberto, G. A., Pacheco, L. R., Gusmão, M. R., \& Gabriel, S. A. (2020). COVID-19 e eventos tromboembólicos. Ulakes Journal Of Medicine, 1.

Rossi, F. H. (2020). Tromboembolismo venoso em pacientes COVID-19. Jornal Vascular Brasileiro, 19.

Soeiro, A. D. M., Leal, T. D. C. A. T., Pereira, M. D. P., Lima, E. G., Figueiredo, A. C. B. D. S., Petriz, J. L. F., \& Serrano, C. V. (2020). Posicionamento sobre Uso de Antiplaquetários e Anticoagulantes nos Pacientes Infectados pelo Novo Coronavírus (COVID-19). 2020. Arquivos Brasileiros de Cardiologia, 115, 292301 .

Tiburi, R. G. B., Jucá, M. C. P., de Souza Sales, M., de Sousa, A. C. G., de Souza, T. N. L., da Silva, C. G. L., \& Gadelha, M. D. S. V. (2021). Coagulopatia induzida pelo estado inflamatório da infecção pela Covid-19. Brazilian Journal of Health Review, 4(2), 8478-8501. 